\title{
Tunable Structural and Optoelectronic Properties of Methylammonium Lead Bromide Perovskite under Pressure
}

\author{
Weizhao Cai*, Rong Zhang and Shanti Deemyad
}

Department of Physics and Astronomy, University of Utah, 115S 1400E, Salt Lake City, Utah 84112, United States

$$
\text { E-mail:wzhcai@physics.utah.edu }
$$

The organic-inorganic lead halide perovskites $\mathrm{MAPbX}_{3}\left(\mathrm{MA}=\mathrm{CH}_{3} \mathrm{NH}_{3}, \mathrm{X}=\mathrm{Cl}, \mathrm{Br}\right.$ and $\left.\mathrm{I}\right)$ have shown superior properties for applications in photovoltaic technology. These materials can be synthesized at a low cost, and have strong solar absorption and excellent power conversion efficiency that currently reaches $\sim 22 \%$. $\mathrm{MAPbBr}_{3}$ is one of these materials, it displays orange colour and with band gap of $\sim 2.3 \mathrm{eV}$ at ambient conditions. The room-temperature structure of $\mathrm{MAPbBr}_{3}$ is cubic with space group $\mathrm{Pm}-3 m$ and shows a diversity of structures during cooling. ${ }^{1-2}$ The framework of all the structures of $\mathrm{MAPbBr}_{3}$ consist of vertex-sharing $\mathrm{PbBr}_{6}$ octahedra, in which the disordered/ordered MA cations occupy the three-dimensional channels and hydrogenbond to the host framework of the $\mathrm{PbBr}_{6}$ octahedra. The high pressure response of structural and electronic changes of $\mathrm{MAPbBr}_{3}$ have been reported recently. ${ }^{3-6}$ We found considerable inconsistencies in these reports and lots of the unsolved issues need to be clarified and explained.

In this work, ${ }^{7}$ we investigated the structural and optoelectronic properties of $\mathrm{MAPbBr}_{3}$ using synchrotron single crystal and powder X-ray diffraction and photoluminescence measurements under various stress conditions. We found the properties of $\mathrm{MAPbBr}_{3}$ can be tuned by employing different pressure-transmitting medium (PTM). While non-hydrostatic compression of $\mathrm{MAPbBr}_{3}$ leads to amorphization above $2.4 \mathrm{GPa}$, under quasi-hydrostatic (Ar) and hydrostatic (He) pressure, the sample remains in crystalline phases. A sequence of phase transitions between two cubic phases and orthorhombic Pnma phase is observed when using Ar, or no PTM. In helium, only transitions between the two cubic structures and a new isostructural phase transition with a large volume collapse to a third cubic phase at $2.7 \mathrm{GPa}$ was observed. The photoluminescence measurements indicate a pressure-induced band gap-narrowing in the cubic phase I, and a blueshift in the orthorhombic structure.

We acknowledge Prof. E. Zurek, T. Bi, N. Zarifi and T. Terpstra for DFT theoretical calculations and C. Zhang and Prof. Z. Valy. Verdeny for providing crystalline samples. We also thank Drs. S. Tkachev, D. Zhang, and J. Smith for experimental support at Advanced Photon Source (APS), Argonne National Laboratory(ANL). The High pressure X-ray diffraction data were collected at HPCAT (Sector 16) and GSECARS (Sector 13) of APS, ANL.

\section{References}

(1) Swainson, I. P.; Hammond, R. P.; Soullière, C.; Knop, O.; Massa, W. J. Solid State Chem. 2003, 176, 97-104.

(2) Knop, O.; Wasylishen, R. E.; White, M. A.; Cameron, T. S.; Oort, M. J. M. V. Can. J. Chem. 1990, 68, 412-422.

(3) Swainson, I. P.; Tucker, M. G.; Wilson, D. J.; Winkler, B.; Milman, V. Chem. Mater. 2007, 19, 2401-2405.

(4) Wang, Y.; Lü, X.; Yang, W.; Wen, T.; Yang, L.; Ren, X.; Wang, L.; Lin, Z.; Zhao, Y. J. Am. Chem.Soc.

2015, 137, 11144-11149.

(5) Jaffe, A.; Lin, Y.; Beavers, C. M.; Voss, J.; Mao, W. L.; Karunadasa, H. I. ACS Cent. Sci. 2016, 2, 201-209.

(6)Kong, L.; Liu, G.; Gong, J.; Hu, Q.; Schaller, R..; Dera, P.; Zhang, D.; Liu, Z.; Yang, W.; Zhu, K. Proc. Natl. Acad. Sci. U. S. A. 2016, 13, 8910- 8915.

(7) Zhang, R.; Cai, W.; Bi, T.; Zarifi, N.; Terpstra, T.; Zhang, C.; Verdeny, Z. V.; Zurek, E.; Deemyad, S., J. Phys. Chem. Lett. 2017, 8, 3457-3465. 